\title{
ANÁLISE DE UMA FANPAGE DO FACEBOOK: PROMOÇÃO DA SAÚDE DE PESSOAS COM FIBROMIALGIA
}

\section{Geovana Barboza Silva Gregório}

Psicóloga, pelo Centro Universitário de Maringá (UniCesumar), Maringá (PR), Brasil. Pesquisadora PIC/ UniCesumar (2016), Maringá, (PR), Brasil.

\section{Mateus Dias Antunes}

Mestre em Promoção da Saúde pelo Centro Universitário de Maringá (UniCesumar); Especialista em Exercício Físico e Reabilitação do Idoso, pela UNIFAMMA, Brasil.

\section{Fabio Ricardo Acencio}

Mestrando no Programa de Pós-graduação em Promoção da Saúde, pelo Centro Universitário de Maringá (UniCesumar), Maringá (PR), Brasil; Bolsista Capes, Brasil.

\section{João Ricardo Nickenig Vissoci}

Duke Global Health Institute, Duke University, Durham, North Carolina.

\section{Leonardo Pestillo Oliveira}

Doutor, Docente do Centro Universitário de Maringá (UniCesumar) no Programa de Pós-graduação em Promoção da Saúde. Pesquisador do Instituto Cesumar de Ciência, Tecnologia e Inovação (ICETI), Brasil.

E-mail: leopestillo@hotmail.com
RESUMO: O presente estudo teve como objetivo analisar a fanpage Grupo de Apoio a Pessoas com Fibromialgia sob uma perspectiva de promoção da saúde, ampliando o conhecimento sobre a síndrome em si. Trata-se de um estudo descritivo-documental, com característica qualitativa. Os dados foram coletados a partir das informações contidas na fanpage. Foram selecionadas três publicações com maior destaque na página de acordo com as características: 1) Postagem com objetivo de divulgação do projeto de extensão; 2) Postagem com objetivo de divulgação científica sobre a fibromialgia; 3) Postagem com objetivo lúdico, com informações não científicas sobre a fibromialgia. A análise dos dados foi realizada por meio da análise de conteúdo das postagens dos usuários da rede. Algumas pessoas comentaram da dificuldade de se encontrar médicos que sejam capacitados para diagnosticar e/ou indicar melhor tratamento para a doença. Conclui-se que o uso das redes sociais como ferramenta de divulgação científica e de promoção da sSaúde é uma vertente importante e que precisa ser aprimorada a cada dia.

PALAVRAS-CHAVE: Síndrome da dor miofascial difusa; Rede social; Qualidade de vida.

\section{ANALYSIS OF A FACEBOOK'S FAN-PAGE: HEALTH ENHANCEMENT OF PEOPLE WITH FIBROMYALGIA}

ABSTRACT: The fan-page Support Group to People with Fibromyalgia is analyzed from the point of view of health promotion and broadening knowledge on the syndrome. The descriptive, documental and qualitative study retrieved data from the fan-page. Three highlighted articles were selected, featuring the following characteristics: (1) dissemination of the extension project; (2) scientific dissemination on fibromyalgia; (3) playful dissemination with non-scientific information on fibromyalgia. Data were analyzed by the Content Analysis of postings by network users. Several people remarked on the difficulty in consulting doctors that would diagnose and indicate the best treatment for the disease. Results show that the use of social networks as tools for scientific dissemination and Health Promotion is highly important and should be improved constantly.

KEY WORDS: Diffuse myofascial pain syndrome; Social network, Life quality. 


\section{INTRODUÇão}

A internet proporciona desde o seu início a possibilidade de compartilhar informações na rede mundial de computadores de maneira mais rápida e prática (ARRUDA; SOUSA; GIRÃO, 2015). Junto a ela, surgem também sites de redes sociais muito populares atualmente, pois fazem parte do cotidiano das pessoas como forma de comunicação mais prática (MICHAEL; MICHAEL; PORCIÚNCULA, 2013).

O conceito de mídia social está presente na cultura há tempos, e corresponde às interações e relações entre as pessoas, em qualquer parte do mundo, fazendo com que a cada ano os responsáveis por sua admiração técnica saibam lidar com as complexidades que surgem a todo instante. No campo da saúde, as mídias sociais ou $m$ Health podem ser usadas para mobilizar as pessoas no processo de ajuda, prevenção de doenças, cuidado dos doentes, e ter a compreensão mais profunda da própria doença (ABBOTT; BARBOSA, 2015).

Considerado um ambiente de trocas de informação, a internet possibilita a participação de pessoas nos mais diversos locais e momentos. Apresentase como um espaço de diálogo, efetividade e de luta de interesses que acaba por propiciar a diminuição de fronteiras entre uma série de indivíduos que podem participar desses espaços, mesmo que seja off-line (GROSSI et al., 2014). O facebook é uma rede social que permite a interação entre os usuários de seu serviço, mas também a exposição de textos, gêneros, discursos e enunciados. É um local propício para análise dos conteúdos atuais que são vinculados pela população em geral na sociedade atual, visto o amplo campo dialógico em que diferentes pessoas se expõem e compartilham seus valores, opiniões, ideologias, alegrias e sofrimentos (MARTORELL; NASCIMENTO; GARRAFA, 2016).

Como forma de divulgação científica, as áreas da saúde também se vinculam a este universo virtual para debater assuntos relacionados à saúde e algumas doenças existentes (FROSSARD; DIAS, 2016). Entre eles a Síndrome da Fibromialgia (SF), que é considerada uma condição reumática progressiva, mas que ainda não apresenta uma fisiopatologia definitiva e nem indicadores mensuráveis da atividade da doença. A principal característica é dor crônica generalizada e, muitas vezes, apresenta sintomas associados como fadiga, problemas do sono e até episódios depressivos (BENNETT et al., 2007; MARTINEZ et al., 2016).

O apoio social é um elemento importante no tratamento da SF, no entanto, não há no Brasil estudos sobre estavariável relacionadaaos sintomas dafibromialgia (FREITAS et al., 2016). Este apoio pode surgir de diversas maneiras e é fundamental na promoção da saúde, sendo recebido de pessoas próximas e profissionais da saúde. Não só no tratamento de doenças, mas o apoio social é essencial na saúde geral e mental (KASHIKAU-ZUCK et al., 2008).

A internet já demonstrou seu potencial quanto ao aspecto educacional e também para a promoção da saúde de pessoas que buscam apoio e informação (DAMÁSIO; NUNES; SOBRAL, 2014). Estudos como os de Moretti et al. (2011) revelam que os usuários de redes sociais, ao buscarem informações sobre alguns fatores específicos, são favorecidos de diversas maneiras, melhorando o relacionamento social, e as questões clínicas, aumento no automanejo da doença, maior qualidade de vida e mudanças comportamentais positivas.

Nesse sentido, abre-se então a possibilidade de que as informações sobre a referida SF sejam veiculadas para que cheguem ao alcance do público interessado. A página do facebook, intitulada Grupo de Apoio a Pessoas com Fibromialgia, surgiu em 2013 como forma de divulgação local de um projeto de extensão universitária homônimo. O objetivo da página foi divulgar o projeto na cidade de Maringá, local onde os encontros ocorrem até o momento, no entanto, com o passar do tempo e a possibilidade de expansão que as redes sociais permitem, a página passou a ser visitada por pessoas de todo o Brasil, iniciando um novo ciclo de divulgação não apenas do projeto, mas também da SF.

O presente estudo se justifica pelo fato de que as mídias sociais, além da enorme gama de informações, são cada vez mais um instrumento de busca de ajuda e troca de experiências, visto que não há fronteiras entre as comunicações e a fanpage do facebook, na qual esta, se torna uma ferramenta que pode viabilizar tal troca de informação, nesse caso sobre a SF, bem como a necessidade de saber mais sobre a doença e de grupos de apoio como o divulgado nessa página. 
O objetivo do presente estudo foi analisar a página Grupo de Apoio a Pessoas com Fibromialgia sob uma perspectiva de promoção da saúde, ampliando o conhecimento sobre a SF em si.

\section{METODOLOGIA}

Este estudo consiste na observação e análise da página Grupo de Apoio a Pessoas com Fibromialgia no facebook. A busca pelas informações foi realizada por meio das publicações presentes na página, para tanto, o facebook foi o facilitador no sentido de registro das mensagens, que ficam salvas na timeline desde quando uma página ou perfil foi criado até a atualidade, o que permite a consulta de postagens e comentários mais antigos. Classifica-se como um estudo qualitativo, pois se utilizaram dados coletados diretamente do que estava postado pelos usuários e seus aspectos subjetivos relacionados ao uso da internet. O estudo pode ainda ser qualificado como descritivo, visto que objetivará maior entendimento de características ainda pouco estudadas pela psicologia (CERVO; BERVIAN; SILVA, 2007).

Foram selecionadas todas as postagens realizadas a partir do ano de 2013 , em que a página foi criada. A seleção das postagens foi realizada a partir de três categorias específicas: 1) postagem com objetivo de divulgação do projeto de extensão; 2) postagem com objetivo de divulgação científica sobre a ibromialgia; 3) postagem com objetivo lúdico, com informações sobre a fibromialgia. Em cada uma das postagens analisouse o conteúdo das mensagens postadas pelos usuários da página, destacando a frequência de participação das pessoas, bem como o conteúdo convergente e divergente existente nas mesmas. Esta seleção foi baseada nos próprios dados fornecidos pelo facebook na página considerada para análise.

Os participantes do estudo não foram entrevistados diretamente, não foram realizadas entrevistas com os usuários da internet, apenas foram analisadas as características de interação destas pessoas com a fanpage do Grupo de Apoio a Pacientes com Fibromialgia, principalmente seu contato direto com comentários a posts, fotos, compartilhamentos e principalmente as mensagens que foram enviadas inbox.

A análise dos dados foi realizada por meio da análise de conteúdo, e se ocupou em analisar as características que possam identificar o perfil das pessoas que interagem com a fanpage do Grupo de Apoio a Pacientes com Fibromialgia, bem como a forma como elas interagem de acordo com o tipo de postagem veiculada. O estudo dispensou aprovação do Comitê de Ética em Pesquisa, pois se trata de uso de dados abertos disponíveis, conforme é preconizado pela resolução do Conselho Nacional de Saúde 466/2012.

\section{RESULTADOS E DISCUSSÃO}

Serão apresentados nesta etapa do estudo os resultados coletados junto às postagens da fanpage. Foram selecionados três tipos de postagens, que serão apresentadas e discutidas a partir da interação dos usuários da rede. Para a seleção das postagens, as informações da própria página foram seguidas como critérios de inclusão; aquelas que tiveram maior alcance de público serão apresentadas aqui.

\subsection{POSTAGEM 1 - OBJETIVO DE DIVULGAÇÃO DO PROJETO DE EXTENSÃO}

Esta publicação foi realizada por um membro do grupo no dia 12/04/2016, com os seguintes dizeres: "As atividades de 2016dogruposerãoiniciadas. Compartilhem e ajudem na divulgação para que possamos ajudar todos os que precisam. O grupo acontecerá no auditório do bloco 6 da Unicesumar em Maringá - Paraná”. 


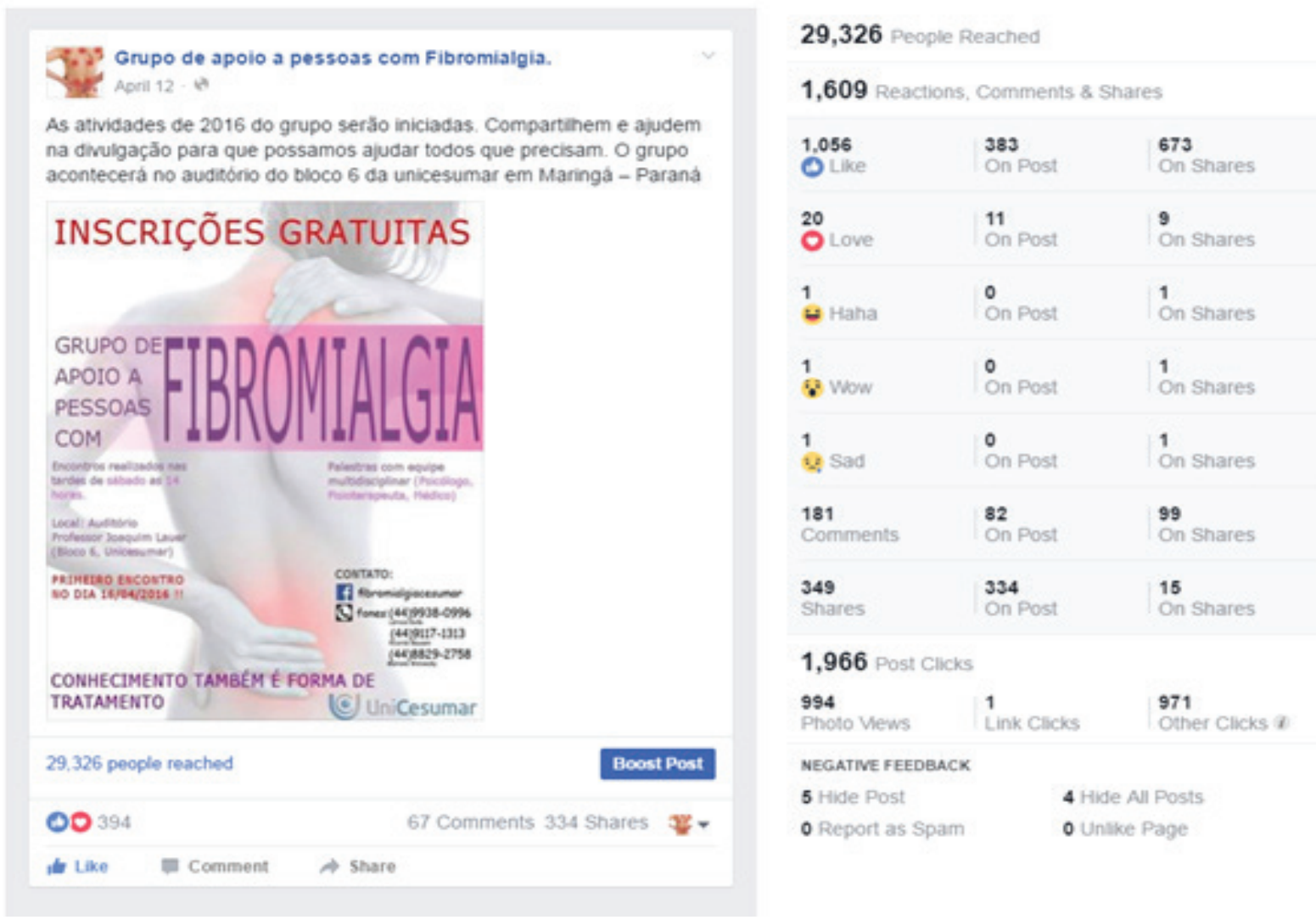

Figura 1. Postagem de divulgação do projeto de extensão

Esta foi uma das primeiras postagens realizadas no ano de 2016 e o objetivo era informar as pessoas, principalmente o público local, do início das atividades práticas do projeto de extensão. Fazia parte do processo de divulgação das ações práticas. Ao todo foram realizados 67 comentários e 334 compartilhamentos nesta postagem.

Com relação aos comentários, muitos eram de pessoas interessadas sobre o local de funcionamento do grupo, solicitando sempre mais informações de como poderiam fazer parte.

Ola meu nome é RFM tenho 38 anos e a 6 fui diagnosticada com fibromialgia Sou de CambÉ PR tenho muito interesse [telefone] Se tivesse algum grupo desses em londrina seria perfeito mais se me incluirem farei o posiível para participar (sic)

Muitas pessoas, principalmente mulheres, tinham interesse em participar dos encontros presenciais, no entanto, eram moradores de outras cidades, inclusive de outros países.

ha mas e no parana eu sou de araraquara sp sofro com fibromialgia a mais de 6 anos nao e facil nao me sinto invalida sem recursos (sic). Oi Boa Noite Muito longe precisamos de grupos Rio Grande do Sul NOVO Hamburgo mais perto sou de Estancia Velha Precisamos de grupos urgente (sic).

Que pena nao ser em Portugal tenho Fibromialgia e avancada tenho dias ela manda mais do que eu ate para comer me vejo aflita (sic).

Durante as postagens dos usuários, muitos começam a conversar entre si e trocar informações. Como ressalta Demiris (2006), dentre os fóruns de discussão, podemos incluir atualmente as redes sociais, em que tem se consolidado como um dos principais canais para troca de informações e apoio de questões referentes aos problemas de saúde. Essas trocas de informações podem ocorrer de diversas maneiras. Muitas pessoas que viram a postagem não emitiram nenhum comentário com relação ao assunto em si, mas "marcaram" outras pessoas que poderiam se interessar por participar do grupo, demonstrando que o alcance das postagens em redes sociais pode ser maior do que o que se imagina. 


\subsection{POSTAGEM 2 - OBJETIVO DE DIVULGAÇÃO CIENTÍFICA SOBRE A FIBROMIALGIA}

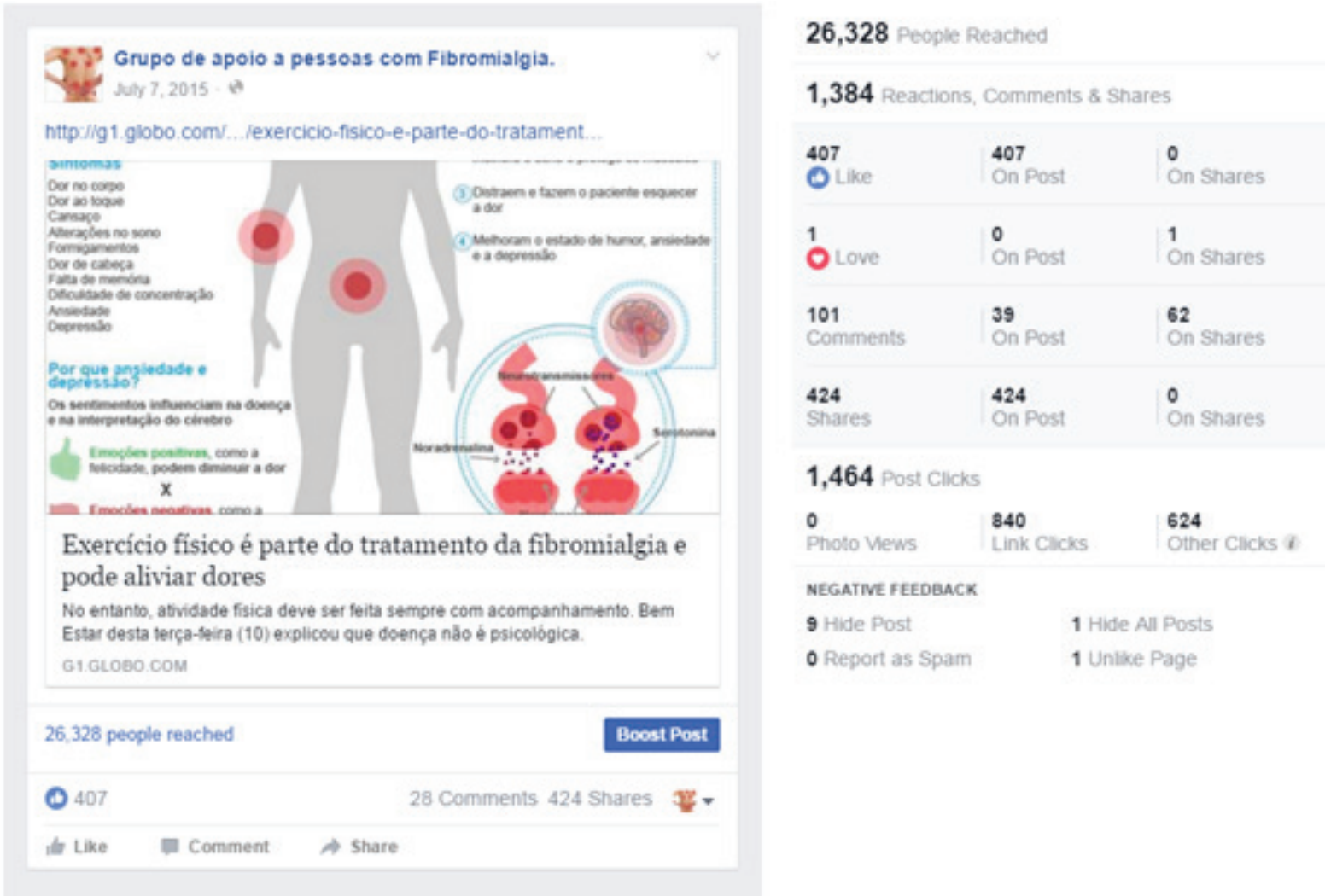

Figura 2. Postagem de divulgação científica sobre a Fibromialgia

A segunda postagem analisada refere-se ao objetivo de transmitir às pessoas algumas informações de cunho científico acerca da fibromialgia. Um dos motivos pelos quais o grupo resolveu realizar postagens com esta característica ocorreu pelo fato de que muitas informações são veiculadas sobre a SF, mas nem todas apresentam caráter científico que possa comprovar sua veracidade. Principalmente no que diz respeito à cura da mesma, algo que a medicina tradicional ainda descarta.

Esta postagem foi realizada por um membro do grupo no dia $07 / 07 / 2015$, sem que houvesse algum tipo de escrita, apenas com o link do site onde as informações mais aprofundadas poderiam ser encontradas: http:// g1.globo.com/bemestar/noticia/2014/06/exercicio-fisicoe-parte-do-tratamento-da-fibromialgia-e-pode-aliviardores.html

Ao todo foram realizados 28 comentários e 424 compartilhamentos nesta postagem. Com relação aos comentários, neste momento algumas pessoas deram depoimentos sobre sua vida com a doença, ou de pessoas próximas. Como a postagem era a respeito da atividade física como uma das formas de tratamento da SF, alguns comentaram sobre suas práticas diárias.

\begin{abstract}
Minha mãe tem fibromialgia porem ela tem artrose nos dois joelhos praticamente não tem mais cartilagem e tbm mtas dores nos ombros e braços No momento da uma simples caminhada ja deixa ela com mtas dores e ela tem dores ate em repouso Para fibromialgia exercícios e bom faz bem Mas e para quem tem fibromialgia e artrose falta de cartilagem e suspeita de hernia de disco Existe alguma atividade física que possa ser feita para o conjunto todo (sic).
\end{abstract}

O comentário anterior apresenta uma das características da fibromialgia, que são as comorbidades, levando muitos pacientes a apresentarem dificuldades de locomoção, quando essas comorbidades são físicas, e em outros casos a dificuldades psicológicas, pois é comum também que alguns problemas psicológicos surjam em conjunto com a mesma. 
Outros comentários referem-se a pessoas que já realizam atividade física e dão seu depoimento sobre como essa prática pode colaborar com o tratamento da doença.

Tenho fibromialgia ha mais de dez anos mas somente ha tres comecei a fazer exercicios com regularidade e frequencia inclusive tenho conseguido ate participar de corridas de 5 e $10 \mathrm{~km}$ coisa que amo fazer Minha qualidade de vida melhorou muito sem contar o alivio que sinto nas dores Exercitar e muito bom e faz bem (sic).

A transmissão de informações científicas é sempre uma alternativa importante para a população em geral. Estudos evidenciaram que o advento da internet favoreceu muito o acesso à informação, produzindo conteúdo massificado e proveniente de diversas fontes (PINTO et al., 2017). Na área da saúde, essa interação via internet, favorece a troca de experiências entre os pacientes e também entre os especialistas da área, colaborando com o manejo de algumas doenças (MURRAY et al., 2005).

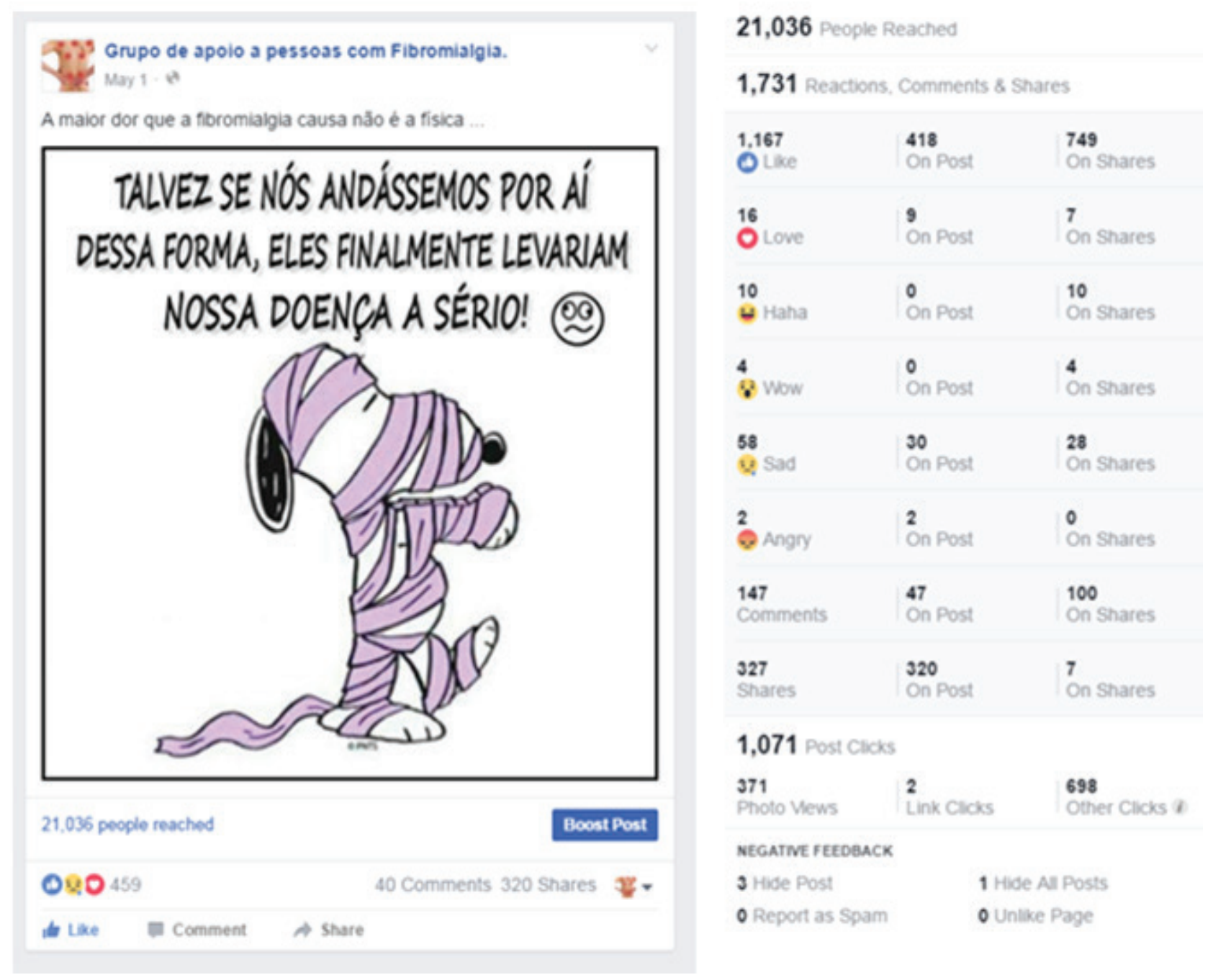

Figura 3. Postagem com objetivo lúdico, contendo informações sobre a fibromialgia 
3.3 POSTAGEM 3 - OBJETIVO LÚDICO, COM INFORMAÇÕES NÃO CIENTÍFICAS SOBRE A FIBROMIALGIA

Esta publicação foi realizada por um membro do Grupo no dia 1\% $105 / 2015$, com os seguintes dizeres: "A maior dor que a fibromialgia causa não é a física ...”.

A terceira postagem analisada demonstra uma das maneiras de falar sobre nossas enfermidades, com o objetivo mais voltado ao lúdico, a intenção era de que as pessoas se identificassem com a imagem e a frase postada. As informações científicas são importantes de serem veiculadas, mas nem sempre o alcance ocorre de forma satisfatória, visto que em alguma medida a linguagem científica não é acessível à população geral.

Ao todo foram realizados 40 comentários e 320 compartilhamentos nesta postagem. Esta postagem apresenta uma quantidade de comentários superior à postagem anterior, que tinha caráter científico. Os comentários foram unânimes na concordância do que foi colocado na postagem, demonstrando que as pessoas que interagiram com a publicação sentiram-se acolhidas pelo que foi postado, concordando então com o fato.

Verdade. Por isto esta doença nos ensina a não se preocupar mais com que os outros vão pensar da gente. Ninguém está no nosso corpo para saber o tamanho de nosso sofrimento. A gente aprende a viver sozinha. A maior dor de quem sofre de fibromialgia e a de saber as pessoas não acreditam no que você está sentindo, se você se arruma um pouco pra saí tem logo alguém dizendo e ainda diz que sofre de dores no corpo, mas só Deus saber a força que faço pra está de bem com a vida mesmo cheia de dor,é difícil. (sic).

Se os de casa tem dificuldade de entende que convive, imagine os de fora. Ja cheguei ao ponto de esperar meu marido sair para trabalhar para voltar a deitar pois as vezes fica difícil suporta as dores. (sic).

O apoio familiar surge como uma variável importante de ser estudada. Alguns estudos já se preocuparam em investigar essa relação, descrevendo o impacto da fibromialgia na funcionalidade e/ou incapacidade e a qualidade de vida dos pacientes. Além disso, Shuster et al. (2009) demonstraram em seu estudo que as mulheres frequentemente relataram menor percepção do apoio familiar, algo que foi descrito por algumas das pessoas que comentaram a postagem sobre a percepção das pessoas sobre a doença.

O uso das redes sociais também pode deixar claro o quanto o contato com outras pessoas pode ser benéfico, inclusive quando o objetivo é fazer com que o outro se sinta bem, com mensagens de apoio e solidariedade.

\begin{abstract}
Por isso somos muito mais que vencedores. Vencemos as dores, os precoceitos e a falta de amor ao proximo vencemos a ignorancia $\mathrm{da}$ existencia de nossas dores. Força meninas (sic).
\end{abstract}

Cada comentário analisado torna-se um ponto importante na vida das pessoas que buscam informações acerca de algumas enfermidades. O uso das redes sociais como ferramenta de promoção da saúde é um caminho em constante mudança, mas que tem gerado resultados positivos no auxílio às pessoas que necessitam.

Este estudo apresenta algumas limitações que merecem destaque. A primeira limitação refere-se ao não contato direto com as pessoas que compartilharam suas informações na fanpage, optou-se em um primeiro momento analisar apenas os comentários de forma aleatória simples, sem aprofundamento sobre o sentimento dos participantes. A segunda limitação do estudo pode ser levantada no sentido de que a fanpage oferece ao administrador da mesma a possibilidade de analisar alguns dados interessantes sobre as postagens, mas neste momento esses dados não foram utilizados.

\section{CONCLUSÃO}

Conclui-se que o uso das redes sociais como ferramenta de divulgação científica e de promoção da saúde é uma vertente importante e que precisa ser aprimorada a cada dia.

\section{AGRADECIMENTOS}

Agradecimento ao Centro Universitário de Maringá (UniCesumar), à Coordenação de Aperfeiçoamento de Pessoal de Nível Superior (Capes) e Instituto Cesumar de Ciência, Tecnologia e Inovação (Iceti), pelo apoio financeiro e concessão de bolsas. 


\section{REFERÊNCIAS}

ABBOTT, P.A.; BARBOSA, S.F.F. Usando tecnologia da informação e mobilização social para combater doenças. Acta Paulista de Enfermagem, v.28, n. 1, p. 3-5, 2015.

ARRUDA, M.P. et al. Divulgação de informações por meio da Internet: serão as redes sociais capazes de reduzir a assimetria informacional entre empresas e investidores?. Revista Evidenciação Contábil \& Finanças, v. 3, n. 2, p. 27-41, 2015.

BENNETT, R.M. et al. An internet survey of 2,596 people with fibromyalgia.BMC Musculoskeletal Disorders, v. 9, n. 1, p. 8-27, 2007.

CERVO, A.L.; BERVIAN, P.A.; SILVA, R. Metodologia científica. 6. ed. São Paulo: Pearson Education do Brasil, 2007.

DAMÁSIO, C.; NUNES, L.; SOBRAL, J.M. A Análise de Redes Sociais no estudo do processo da construção da ajuda mútua da pessoa com doença oncológica com blogue. REDES, v. 5, n. 1, p. 153-189, 2014.

DEMIRIS, G. The diffusion of virtual communities in health care: Concepts and challenges. Patient Education and Counseling, v. 62, n. 2, p. 178-188, 2006.

FREITAS, R.P. Impacto do apoio social sobre os sintomas de mulheres brasileiras com fibromialgia. Revista Brasileira de Reumatologia, v. 56, n. 1, p. 1-7, 2016.

FROSSARD, V.C.; DIAS, M.C.D. O impacto da internet na interação entre pacientes: novos cenários em saúde. Interface - Comunicação, Saúde, Educação, v. 20, n. 57, p. 349-361, 2016.

GROSSI, M.G.R. et al. A utilização das tecnologias digitais de informação e comunicação nas redes sociais pelos universitários brasileiros. Texto Digital, v. 10, n. 1, p. 4-23, 2014.

KASHIKAR-ZUCK, S. Family factors, emotional functioning, and functionalimpairment in juvenile fibromyalgia syndrome. Arthritis \& Rheumatology, v. 59, n. 10, p. 1392-1398, 2008.

MARTINEZ, J.E. et al. Avaliação de parâmetros clínicos de pacientes com Fibromialgia após 5 anos de evolução. Acta Fisiátrica, v. 8, n. 2, p. 71-74, 2016.

MARTORELL, L.B.; NASCIMENTO, W.F.; GARRAFA, V. Redes sociais, privacidade, confidencialidade e ética: a exposição de imagens de pacientes no facebook. Interface - Comunicação, Saúde, Educação, v. 20, n. 56, p. 13-23, 2016.

MICHEL, M.; MICHEL, J.; PORCIÚNCULA, C.G. A Comunicação organizacional, as redes sociais e seus desafios: afetos e emoções nesse contexto. Revista Internacional de Direitos Humanos, v. 3, n. 6, p. $117-$ 136, 2013.

MORETTI, F.A. et al. Avaliação do nível de conhecimento sobre fibromialgia entre usuários da internet. Revista Brasileira de Reumatologia, v. 51, n. 1, p. 7-19, 2011.

MURRAY, E. et al. Interactive health communication applications for people with chronic disease. The Cochrane Database of Systematic Review, v. 19, n. 4, p. 4274-4285, 2005.

PINTO, A.C.F. et al. Uso de tecnologias da informação e comunicação na educação em saúde de adolescentes: revisão integrativa. Revista de Enfermagem da UFPE, v. 11, n. 2, p. 634-644, 2017.

SHUSTER, J. et al. Understanding the psychosocial profile of women with fibromyalgia syndrome. Pain Research and Management, v. 14, n. 3, p. 239-245, 2009.

Recebido em: 2017-06-20 Aceito em: 2017-12-01 